\title{
Fulminanter Nutzenzuwachs in der Neurologie (Teil 1)
}

Hans H. Jung, Zürich; Marcel Arnold, Bern; R. Du Pasquier, Lausanne; Andreas F. Hottinger, Lausanne; Georg Kägi, St. Gallen; Ludwig Kappos, Basel; Philipp Lyrer, Basel; Roland Martin, Zürich; Patrick Roth, Zürich; Michael Weller, Zürich

In der Neurologie kam es in den letzten 20 Jahren zu grundlegenden, teilweise revolutionären Veränderungen der Behandlungskonzepte und -erfolge. Krankheiten wie Schlaganfall, Multiple Sklerose und Hirntumoren, die früher unbehandelbar waren, können nun mit neuen Therapiemethoden angegangen werden. Dies verbessert die Lebenserwartung und Lebensqualität der Betroffenen markant und reduziert Invaliditätskosten.

\section{Trotz Schlaganfall reale Chancen auf ein Leben ohne alltagsrelevante Behinde- rungen}

Jedes Jahr erleiden etwa 16000 Personen in der Schweiz, etwa ein Drittel davon noch im Erwerbsalter, einen Schlaganfall. Auch wenn Schlaganfälle immer noch eine der häufigsten Ursachen für Tod und Behinderung sind, haben sich die Chancen, einen Hirnschlag ohne oder mit nur geringen Einschränkungen zu überleben, in den letzten Jahrzehnten deutlich verbessert.

Eine 60-jährige Patientin wird mit Koma in eine Schlaganfallstation gebracht, wo ein Blutgerinnsel in einer Hirnarterie festgestellt wird (Basilaristhrombose). Nachdem ihr Medikamente zur Auflösung des Gerinnsels verabreicht wurden (systemische Thrombolyse) wird sie ins Schlaganfallzentrum weiterverlegt. Dort kann mit Hilfe eines durch das Gefässinnere zur verschlossenen Stelle geleiteten Katheters derThrombus entfernt werden (endovaskuläre Thrombektomie). Die Patientin verlässt wenig später das Spital ohne bleibende Beeinträchtigungen.

Diese Situation, die vor 20 Jahren fast sicher zum Tode der betroffenen Patientin geführt hätte, zeigt exemplarisch den Fortschritt der Schlaganfallbehandlung: (1) Die systemische Thrombolyse hat sich flächendeckend etabliert. (2) Die endovaskuläre Thrombektomie wird in geographisch sinnvoll verteilten Schlaganfallzentren rund um die Uhr angeboten, und (3) durch die Organisation in Schlaganfallnetzwerken können mehr Patienten eine spezialisierte Behandlung erhalten. So kann bei einem schweren Schlaganfall nach systemischer Thrombolyse kombiniert mit endovaskulärer Therapie ein Leben ohne alltagsrele- vante Behinderung bei etwa der Hälfte der Patienten erreicht werden.

\section{Mit Multipler Sklerose (MS) jahrzehnte- lang normal leben}

In der Schweiz sind 10000 bis 15000 Menschen von Multipler Sklerose betroffen, einer Erkrankung, die häufiger Frauen betrifft als Männer und deren erste Symptome meist zwischen dem 20. und 40. Lebensjahr auftreten. Abhängig davon, wo die meist schubweise auftretenden Entzündungsherde im zentralen Nervensystem liegen, können Symptome wie Sehstörungen, Probleme der Motorik oder Koordination, Schmerzen sowie psychische oder kognitive Beeinträchtigungen auftreten.

Die Multiple Sklerose hat nach wie vor einen sehr erschreckenden Ruf, und wenn Patienten mit dieser Diagnose konfrontiert werden, sehen sie sich in der Regel schon in Bälde im Rollstuhl sitzen. Dies entspricht aber nicht mehr den Tatsachen. Auch wenn die MS immer noch nicht heilbar ist, kam es in den letzten Jahren $\mathrm{zu}$ einer dramatischen Verbesserung der Therapiemöglichkeiten. Aktuell können wir, dank rund einem

Auch wenn die neuen MS-Therapien kostspielig sind, erlauben sie in hohem Masse, indirekte Kosten zu vermeiden.

Dutzend verschiedener neuer Therapien, den Verlauf dieser Krankheit entscheidend verlangsamen, so dass die überwiegende Mehrzahl der MS-Patienten über 
Jahrzehnte hinweg ein völlig normales Leben führen, Kinder grossziehen und Vollzeit arbeiten können. Diese Entwicklung ist nicht nur für die Betroffenen und ihre Neurologen höchst befriedigend. Darüber hinaus haben die spektakulären Behandlungsfortschritte auch signifikante positive volkswirtschaftliche Konsequenzen, weil die in der Regel jungen MSPatienten ohne Unterstützung leben und auch erwerbstätig sein können. Sie benötigen weniger pflegerische Unterstützung sowie Hilfsmittel und beziehen auch weniger Rentenleistungen. Auch wenn die neuen MS-Therapien kostspielig sind, erlauben sie in hohem Masse, indirekte Kosten zu vermeiden.

\section{Gezieltere Behandlungen von Hirntumoren}

Korrespondenz:

Schweizerische Neuro-

logische Gesellschaft (SNG)

c/o IMK

Münsterberg 1

CH-4001 Basel

Kontaktadressen der verantwortlichen Co-Autoren Prof. Dr. med. Hans H. Jung Vizepräsident SNG

Klinik für Neurologie UniversitätsSpital Zürich Frauenklinikstrasse 26 CH-8091 Zürich hans.jung[at]usz.ch

Dr. Georg Kägi Neurologische Klinik Kantonsspital St. Gallen Rorschacher Strasse 95 CH-9007 St. Gallen georg.kaegi[at]kssg.ch

Prof. Dr. Renaud Du Pasquier Service de neurologie CHUV, BHO7

Rue du Bugnon 46

CH-1001 Lausanne renaud.du-pasquier[at] chuv.ch

Prof. Dr. Michael Weller Klinik für Neurologie UniversitätsSpital Zürich Frauenklinikstrasse 26 CH-8091 Zürich michael.weller[at]usz.ch
Hirntumoren treten in der Schweiz jedes Jahr bei etwa 600 Personen auf und führen bei vielen zum Tod. Da Hirntumoren oft bereits im frühen Lebensalter auftreten, gehören sie zu den Krebserkrankungen, die am meisten verlorene Lebensjahre verursachen.

Neue Methoden der Hochdurchsatzdiagnostik haben das Verständnis der molekularen Krankheitsentstehung und damit die Klassifikation von Hirntumoren in den letzten 10 bis 20 Jahren drastisch verändert. So ist es möglich abzuschätzen, ob eine Therapie für einen $\mathrm{Pa}$ tienten nicht erfolgversprechend ist, so dass ihm belastende Therapieversuche und Nebenwirkungen wie Übelkeit, Blutbildveränderungen und Infektgefahr erspart werden können. Durch die bessere Kenntnis der Tumorbiologie können aber auch aggressive Tumorerkrankungen rascher erkannt und besser zielgerichtet behandelt werden. Grosse Fortschritte sind auch im Bereich der Sicherheit und Präzision von neurochirurgischer und radioonkologischer Behandlung erzielt worden. Der Einsatz der medikamentösen Therapie führt heute z.B. bei Kleinhirntumoren von Kindern oder bei Tumoren des Lymphgewebes des zentralen Nervensystems zumindest bei Subgruppen von Patienten zu einer Heilung, die vor wenigen Jahren noch für unmöglich gehalten wurde. Auch bei anderen, weiterhin unheilbaren Erkrankungen wie dem Glioblastom, dem häufigsten, bösartigen Hirntumor bei Erwachsenen, steigt die Zahl von Langzeitüberlebenden. Wesentlicher Schritt der Verbesserung der Prognose und Lebensqualität von Patienten mit Hirntumoren war die Einführung multidisziplinärer Strukturen zur Optimierung der Diagnostik und Therapie.

\section{Fazit: ein fulminanter Nutzenzuwachs}

Die Fortschritte in der klinischen Neurologie der letzten Jahre - hier dargestellt an den Beispielen von Schlaganfall, Multipler Sklerose und Hirntumoren - sind das Resultat intensiver Forschung und stellen eine Erfolgsgeschichte für die Patienten dar. Für die Zukunft ist abzusehen, dass für viele, bislang nicht kausal therapierbare neurologische Erkrankungen wie Amyotrophe Lateralsklerose (ALS), Demenzerkrankungen oder genetisch bedingte neurologische und neuromuskuläre Erkrankungen spezifische Therapiemethoden zur Verfügung stehen könnten. Die Kosten dieser Entwicklung sind hoch, sind aber in einer Gesamtrechnung mit einer oft markant verbesserten Lebensqualität und verlängerter Lebenszeit zu sehen. Neben diesem Nutzen für die individuellen Patienten und ihr Umfeld erzielen die neuen Therapien auch einen ökonomischen Nutzen, weil oftmals z.B. Behinderungen und Berentungen vermieden oder verzögert werden und Pflegebedarf reduziert wird.

\section{Zusammenfassung}

Die Fortschritte der Neurologie haben in den letzten 20 Jahren zwar Behandlungskosten erhöht, aber auch einen erheblichen Mehrwert für die Patienten gebracht. So ermöglichen Schlaganfallbehandlungen heute deutlich mehr Menschen ein Leben ohne alltagsrelevante Behinderungen. Auch die dramatisch verbesserten Therapien bei Multipler Sklerose ermöglichen vielen Patienten über Jahrzehnte hinweg ein völlig normales Leben. Behandlungsentscheide bei Patienten mit Hirntumoren können heute auf einer verbesserten Grundlage teilweise mit Heilungsaussicht - getroffen werden. Diese Fortschritte geben den Betroffenen Lebensqualität und Lebenszeit - und sparen der Gesellschaft, unter anderem, hohe Invaliditätskosten. 